\title{
Genome-wide gene expression profiling and mutation analysis of Saudi patients with Canavan disease
}

Namik Kaya, $P h D^{1}$, Faiqa Imtiaz, $P h D^{1,2}$, Dilek Colak, $P h D^{3}$, Moeenaldeen Al-Sayed, $\mathrm{MD}^{4}$, Ali Al-Odaib, $P h D^{1}$, Fatma Al-Zahrani, BSc ${ }^{1}$, Bashayer R. Al-Mubarak, BSc ${ }^{1,2}$, Mohammad Al-Owain, $\mathrm{MD}^{4}$, Hesham Al-Dhalaan, $\mathrm{MD}^{5}$, Aziza Chedrawi, $M D^{5}$, Zuhair Al-Hassnan, $M D^{4}$, Serdar Coskun, $\mathrm{PhD}^{6}$, Nadia Sakati, $\mathrm{MD}^{4}$, Pinar Ozand, MD, $P h D^{1,7}$, and Brian F. Meyer, $P h D^{1,2}$

\begin{abstract}
Purpose: Canavan disease, caused by a deficiency of aspartoacylase, is one of the most common cerebral degenerative diseases of infancy. The aims of this study were to identify the mutations associated with Canavan disease in Saudi Arabia and to identify differentially expressed genes likely to contribute to the development of this disease. Methods: Polymerase chain reaction, long polymerase chain reaction, multiplex ligation-dependent probe amplification, sequencing, array comparative genomic hybridization (aCGH), and global gene expression profiling were used to determine putative mutations and likely gene signatures in cultured fibroblasts of patients from Saudi Arabia. Results: One novel and one known large deletion and two previously known mutations (IVS4 $+1 \mathrm{G}>\mathrm{T}$ and G27R) were identified. Compared with controls, 1440 genes were significantly modulated in Canavan patients (absolute fold change $[F C] \geq 4$ ). Genome-wide gene expression profiling results indicated that some genes, involved in apoptosis, muscle contraction and development, mitochondrial oxidation, inflammation and glutamate, and aspartate metabolism, were significantly dysregulated. Conclusions: Our findings indicate that the presence of muscle weakness and hypotonia in patients may be associated with the dysregulated gene activities of cell motility, muscle contraction and development, actin binding, and cytoskeletal-related activities. Overall, these observations are in accordance with previous studies performed in a knockout mouse model. Genet Med 2008:10(9):675-684.
\end{abstract}

Key Words: Canavan disease, genome-wide gene expression, muscle weakness and hypotonia, large novel deletions, aCGH, MLPA, long-PCR, mtDNA sequencing

Canavan disease (CD) or Canavan-Van Bogaerrt-Bertrand disease (OMIM 271900) is a spongiform encephalopathy caused by the deficiency of the cytosolic enzyme aspartoacylase (ASPA, EC 3.5.1.15), which subsequently causes the accumulation of $\mathrm{N}$-acetylaspartic acid (NAA) in urine and in the central nervous system. ${ }^{1}$ This disease is common in Jews of Ashkenazi origin ${ }^{2}$ and is one of the most common metabolic errors in Saudi Arabia. ${ }^{3}$

Human ASPA is located on the short arm of chromosome 17 (17p13ter) and spans approximately 23,405 bp, comprises six ex-

From the ${ }^{1}$ Department of Genetics; ${ }^{2}$ Arabian Diagnostic Laboratories; ${ }^{3}$ Departments of BESC; ${ }^{4}$ Medical Genetics; ${ }^{5}$ Neurosciences; and ${ }^{6}$ Pathology, King Faisal Specialist Hospital and Research Center, Riyadh, Kingdom of Saudi Arabia.

Namik Kaya, PhD, NeuroGenetics Research Unit, Department of Genetics, KFSHRC, P.O. Box 3354, MBC 03, Riyadh 11211, Kingdom of Saudi Arabia. E-mail: nkaya@kfshrc.edu.sa; namikkaya@gmail.com.

Pinar Ozand is currently at Pakize Tarzi Maternity and Children's Hospital, Clinica, Yildiz Posta Caddesi, Evren Sitesi, C-D Blok, No: 34, Levent, Istanbul, 34349, Turkey.

The first two authors contributed equally to this work.

Disclosure: The authors declare no conflict of interest.

Supplementary figures are available via the ArticlePlus feature at www.geneticsinmedicine.org.

Submitted for publication March 9, 2008.

Accepted for publication June 10, 2008.

DOI: 10.1097/GIM.0b013e31818337a8 ons, and encodes 313 amino acids. ${ }^{4}$ ASPA is a metalloprotease; a zinc containing carboxypeptidase. Studies performed by Herga et al. and Bitto et al. 5,6 have identified the zinc binding ligands and the putative catalytic site. The triad of His21, Glu24, and His116 perfectly form a niche where zinc is located. ${ }^{6}$ A Glu178 is located in the vicinity of the zinc metal site and has been considered to be the active site of the enzyme. The inhibition of the native enzyme by two zinc-chelating agents EDTA and 1,10-phenanthroline, and by the histidine specific reagent DEPC, strongly confirms that ASPA is a zinc metalloenzyme. $^{6}$

ASPA shows remarkable similarity to carboxypeptidase A and probably functions in a similar manner that is by a "promoted-water pathway." It is hypothesized that GLU178 behaves as a general base and activates a water molecule kept appropriately in coordination with zinc at the active site. ${ }^{5}$ The structure of its carboxy-terminal does not alter other protein degradation pathways except for $N$-acetylaspartate. ${ }^{5}$ This is important since ASPA is a cytosolic enzyme and apparently acts only as a specific peptidase. The C-domain also forms a pocket that can accommodate only NAA. ${ }^{5}$ In the mouse gene knockout model, it has been shown that ASPA does not hydrolyze another abundant brain peptide $N$-acetyl-aspartyl-gluta- 
mate nor does it compete with glutamate carboxypeptidase II activity. $^{7}$

Bitto et al. ${ }^{5}$ conducted careful crystallographic protein analysis in rat and human ASPA. They have classified the ASPA mutations into five groups: those that affect (1) zinc coordination, (2) hydrogen bonding, (3) hydrophobic core, (4) premature termination, and (5) destabilization of the dimer surface. Also, a model-based study by Hershfield et al. ${ }^{8}$ indicated that the mutations at the putative zinc-binding residues (H121G, E24/D/G, and H116G0), the general proton donor (E178A), and mutants that switch over the order of zinc-binding residues (H121E/E24H and E24H/H16E) produced full proteins with no ASPA activity. Mutations at the carboxyl terminal (R71N) also lead to undetectable ASPA activity. Most mutations found in the patients lead to a severe phenotype and undetectable ASPA activity. Only rare mutations such as G212A lead to a milder phenotype compatible with a prolonged life span. ${ }^{9}$

$\mathrm{CD}$ in Ashkenazi Jews is associated with two point mutations: E285A and Y231X, whereas different point mutations are found in non-Jewish patients of European descent. ${ }^{4,10,11}$ In both ethnic groups, certain patients have unusual mutations. In a non-Jewish population in New York, Zeng et al. ${ }^{12,13}$ found 14 novel mutations including three deletions 923delT, 33del13, and 244delA. In non-Jewish patients, Kaul et al. ${ }^{11}$ found a 1 bp deletion (32delta T) and 4 bp deletion (8768AGAA).

Various studies to elucidate the pathophysiology of CD have been performed, and it has been reported that the NAA is synthesized in neurons probably serving as a rapidly functioning water pump that effluxes water against a gradient. The NAA then enters into oligodendrocytes where ASPA deacetylases it. When NAA is present in excess, mitochondrial swelling occurs. ${ }^{14,15}$ However, it is still not clear why this mitochondrial changes occurs. Demyelination is a constant feature of this disease and accounts for the clinical appearance of early megalencephaly, severe pyramidal tract lesions, blindness, and early death.

In this study we describe mutation analysis of CD patients in Saudi Arabia and present genome-wide gene expression profiles performed on total RNA isolated from cultured fibroblasts of two Saudi CD patients. We compared our gene expression findings with the previous studies performed in the brain of CD knockout mouse by Surendran et al. ${ }^{16-18}$ Our genomewide expression studies showed many other critical changes in genes involved in glutamate and aspartate metabolism, cytoskeletal processes, cell motility-related activities, different growth factor signaling pathways, axon guidance pathways, muscle contraction and development, and mitochondrial oxidation were significantly affected. We report in the Saudi population the presence of two previously identified single point mutations (splice site and missense mutations), a novel exon 3 deletion (3346 bp), and a previously reported $92 \mathrm{~kb}$ large deletion completely abolishing the presence of ASPA and most of the adjacently located TRVP3 gene.

\section{PATIENTS AND METHODS}

\section{Patients}

Five patients from different parts of Saudi Arabia were included in this study. The patients were from unrelated families and were clinically and biochemically diagnosed with CD using standard criteria confirming this disorder. The project was approved by King Faisal Specialist Hospital and Research Centre's Internal Review Board and informed consent was given by all individuals participating in this study.

\section{Blood collection and fibroblast culture}

The collection of blood and establishment of fibroblasts from skin biopsies were as previously described. ${ }^{3}$

\section{DNA isolation}

Five milliliters of whole blood from consented patients was collected into EDTA tubes for genomic DNA isolation. The DNA was isolated from the blood using the PureGene DNA Purification Kit according to the manufacturer's instructions (Gentra Systems, Inc., Minneapolis, MN). QIAGEN's Mini DNA isolation Kit (QIAGEN, Valencia, CA) was used to isolate DNA from cultured cells. The DNA was checked on a standard agarose gel and then quantified spectrophotometrically. Good quality DNA was stored in aliquots at $-20^{\circ} \mathrm{C}$ until further use.

\section{Long polymerase chain reaction}

About 100-200 ng DNA was used as a template for longpolymerase chain reaction (PCR) amplification using Extensor High-Fidelity PCR Enzyme and PCR master mix (ABgene Inc., Rochester, NY). The long-PCR was performed according to the manufacturer's recommendations (ABgene Inc.). Several primer sets spanning the regions from intron 2 to 3 were designed to detect the deletion spanning exon 3 and cover the breakpoints of the deletion.

\section{Polymerase chain reaction}

DNA was amplified by PCR using intronic primers designed to amplify the coding exons of ASPA and TRPV3. We used the UCSC Genome Browser (http://genome.ucsc.edu), NCBI Entrez (http://www.ncbi.nlm.nih.gov/sites/gquery), and Ensembl database (http://www.ensembl.org) to obtain exon and intron boundaries. ExonPrimer (http://ihg.gsf.de/ihg/ExonPrimer. html) and Primer3 (http://frodo.wi.mit.edu/cgi-bin/primer3/ primer3www.cgi) were used to design primers for each exon including exon/intron boundaries. PCR was performed using the DNA Engine PTC-200 (MJ Research, Watertown, MA) in a final volume of $20 \mu \mathrm{L}$ containing approximately $10 \mathrm{ng}$ of genomic DNA, $50 \mathrm{mM} \mathrm{KCl}, 10 \mathrm{mM}$ Tris- $\mathrm{HCl}$ (pH 8.3), 1.5 $\mathrm{mM} \mathrm{MgCl} 2,100 \mu \mathrm{M}$ deoxyribonucleotide triphosphates (dNTPs), 1 unit of HotStar Taq polymerase (QIAGEN), and 50 ng of each primer. Thermocycling consisted of an initial denaturation at $95^{\circ} \mathrm{C}$ for 15 minutes followed by 35 cycles of PCR. Each cycle of PCR consisted of denaturation at $94^{\circ} \mathrm{C}$ for 60 seconds, annealing at $62-68^{\circ} \mathrm{C}$ for 60 seconds, and extension at $72^{\circ} \mathrm{C}$ for 60 seconds. A final extension step of 10 minutes at 
$72^{\circ} \mathrm{C}$ was added. All primer sequences used for PCR, longPCR, RT-PCR, and sequencing experiments are available on request.

\section{Mutation detection and analysis}

Direct sequencing was performed on either ABI 3100 Automated DNA Sequence Analyzer (Applied Biosystems, Foster City, CA) or MegaBACE 1000 DNA Analysis System (Molecular Dynamics, Sunnyvale, CA) according to the manufacturer's recommendations. Briefly, purified PCR products or longPCR products were used for sequencing reactions using DNA Dye Terminator Cycle Sequencing Kit (Applied Biosystems) and purified with Dye-Ex protocol before it was run on ABI 3100 Automated DNA Sequence Analyzer. For the MegaBACE 1000, sequencing reactions were performed using an Amersham ET Dye Terminator Cycle Sequencing Kit (Amersham Biosciences, Piscataway, NJ) following the manufacturer's instructions. Sequencing reactions were desalted and unincorporated nucleotides removed using ethanol precipitation and resuspended in a formamide EDTA solution for injection on a MegaBACE 1000 DNA Analysis System (Molecular Dynamics).

Sequence data were blasted against the NCBI database and aligned with the reference sequences. Sequencing analysis on all coding exons, exon/intron junctions of ASPA, and contigs assembled using Lasergene-SeqMan version 6.1 (DNA Star, Inc., WI) and ChromasPro 1.31 (Technelysium Pty Ltd., Australia) softwares. Genbank (accession no. AF376770) was used as reference sequence. Numbering commenced with the A of the ATG initiation codon as +1 .

\section{mtDNA sequencing}

DNA isolated from cultured fibroblasts or whole blood was used for PCR amplification of mtDNA. The entire coding region of the mitochondrial genome was amplified in 24 separate PCRs using common cycling conditions as detailed elsewhere ${ }^{19}$ for the patients, certain family members, and controls. Each successfully amplified fragment was directly sequenced using the BigDye Terminator V3.1 Cycle Sequencing kit (Applied Biosystems), and samples were run on the ABI Prism 3100 Sequencer (Applied Biosystems).

\section{Sequence analysis of the mitochondrial DNA coding region}

Sequencing results were compared with the corrected Cambridge reference sequence. ${ }^{20}$ All fragments were sequenced in both forward and reverse directions at least twice for confirmation of any detected variant. Sequence results were compared with the MITOMAP database. ${ }^{20}$

\section{Multiplex ligation-dependent probe amplification}

The multiplex ligation-dependent probe amplification (MLPA) technique (SALSA ${ }^{\circledR}$ P025-Canavan MLPA; MRCHolland, Amsterdam, Holland) was performed in patient samples for which mutations were not identified using standard sequencing analysis. ${ }^{21}$ Briefly, in each reaction 250-300 ng of genomic DNA was denatured and hybridized with the SALSA ${ }^{\circledR}$ probe-mix. After probe hybridization, a ligation reaction was performed using ligase- 65 enzyme and subsequent products were amplified by 35 cycles of PCR; 30 seconds at $95^{\circ} \mathrm{C}, 30$ seconds at $60^{\circ} \mathrm{C}, 1$ minute at $72^{\circ} \mathrm{C}$, and a final extension step of 20 minutes at $72^{\circ} \mathrm{C}$. The PCR products were separated and analyzed using an ABI-3130xl Genetic Analyzer (Applied Biosystems). Peak heights for each fragment were visually and quantitatively compared with those of a normal control sample and deletions were suspected when the peak intensities differed by more than $30 \%$. To ensure accurate analysis of suspected deletions of ASPA, normalization of peak areas of each amplification product was performed by normalization methods described in the manufacturer's protocol.

\section{aCGH experiments and data analysis}

We used the Human Genome CGH Microarray Kit 244A (Agilent Technologies, Santa Clara, CA) according to the manufacturer's instructions with minor modifications. Briefly, 3 $\mu \mathrm{g}$ of genomic DNA was digested overnight with AluI and RsaI followed by purification. A universal human male genomic DNA (Promega, Madison, WI) was used as reference in the hybridization. The Bioprime Labeling Kit (Invitrogen Inc., Carlsbad, CA) was used to label all of the digested patient's DNA and reference DNA separately with Cy5-dUTP and Cy3dUTP (Perkin-Elmer, Waltham, MA), respectively. Labeled patient DNA and reference DNA were mixed, purified, and prepared for hybridization. After 38 hours of hybridization, repeated wash cycles were performed according to the manufacturer's instructions. The slides were scanned on an Agilent DNA Microarray Scanner (Agilent Technologies) and images were extracted using the manufacturer's extraction software. The generated QC report was analyzed to ensure proper hybridization and grid placement. The images and data generated by the feature extraction tool were loaded into CGH Analytics 3.4.40 software (Agilent Technologies) to allow visualization of the data. The default settings of the CGH Analytics for the identification of chromosomal aberrations were applied. Zscore algorithm with a threshold of 5.0 was chosen with the 60 pt window to see the distribution of data points and to identify chromosomal aberrations.

\section{RNA isolation}

Cultured fibroblasts were used for total RNA isolation using a QiaAmp RNA Blood Mini Kit (QIAGEN). Total RNA quality and quantity was determined by measuring absorbance spectra on a UV/Vis spectrophotometer (NanoDrop ${ }^{\circledR}$ ND-1000; Nanodrop Inc., Wilmington, DE) and further analyzed using the RNA 6000 Nano Assay and 2100 Bioanalyzer (Agilent Technologies). The undegraded, high-quality RNA was either immediately used in the experiments or stored at $-80^{\circ} \mathrm{C}$ for further use.

\section{ABI 1700 microarray experiments}

Total RNA $(2 \mu \mathrm{g})$ was subjected to first strand cDNA synthesis, cycled for 10 minutes at $25^{\circ} \mathrm{C}, 2$ hours at $42^{\circ} \mathrm{C}$, and 15 minutes at $0^{\circ} \mathrm{C}$. The newly synthesized first strand cDNA was subjected to a second strand synthesis using RNAse-H and 
Escherichia coli DNA polymerase by incubating the cDNA at $16^{\circ} \mathrm{C}$ for 2 hours. Double-stranded cDNA was purified and approximately $150 \mu \mathrm{L}$ of cDNA was used in in vitro transcription reaction. During the in vitro transcription reaction DIGUTP was incorporated into cRNA. Labeled cRNA $(10 \mu \mathrm{g})$ was purified, quantified, and hybridized to the Human Genome Array V.2 for 16 hours (Applied Biosystems). After washing and rinsing of the arrays, chemiluminescent enhancement was performed on the arrays. Each array was read using the ABI 1700 Chemiluminescent Microarray Analyzer (Applied Biosystems). Applied Biosystems' Expression Analysis Software was used for primary analysis. Two technical replicates were performed on each sample and controls.

\section{Microarray data analysis and functional annotation of genes}

Images were autogridded and the chemiluminescent signals were quantified, background subtracted, and finally, spot- and spatially normalized using the Applied Biosystems 1700 Chemiluminescent Microarray Analyzer software v 1.1.1. For transcriptome analysis, detection thresholds were used following the manufacturer's recommendations. Detection threshold was set as $\mathrm{S} / \mathrm{N}>3$ and quality flag $<5000$. Two technical replicates were performed on each sample for the controls, Patients 4 and 5, for a total of eight microarrays.

To determine gene expression differences, the open source and open development software project $\mathrm{R}$ together with the Bioconductor packages were used. ${ }^{22}$ Probability $(P)$ values were adjusted for multiple comparisons using false discovery rate approach. The $t$ test was performed if the probe showed $\mathrm{S} / \mathrm{N}>3$ in $50 \%$ or more of the samples in either group. It has been shown that for low sample sizes, using the fold change to identify differentially expressed genes has been shown to outperform other $t$ statistic methods, ${ }^{23}$ which are due to the fact that these methods provide less reliable estimate of the variance because of the small sample size. Hence, to determine the differentially expressed genes we used a stringent criteria of absolute fold change $\geq 4$ between the disease and normal control for the probes showing $\mathrm{S} / \mathrm{N}>3$ in $50 \%$ or more of the samples in either group. Kmeans clustering to group differentially expressed genes and hierarchical clustering samples using Eucledean distance with average linkage clustering was performed by using TIGR Multi Experiment Viewer (MeV4.0) (available at www.tm4.org).

Functional annotation and biological-term enrichment analysis was performed by using DAVID Bioinformatics Resources, ${ }^{24}$ Expression Analysis Systematic Explorer (EASE), ${ }^{25}$ and Protein ANalysis THrough Evolutionary Relationships (PANTHER) Classification System. ${ }^{26,27}$ Over-representation was defined by $P<0.05$.

\section{RESULTS}

\section{Patient descriptions}

\section{Patient 1}

This patient presented at 9 months of age with progressive neuroregression and macrocephaly. Currently, at the age of 3 years 5 months, he has spastic quadreperesis, central hypotonia, oropharyngeal dysplasia, macrocephaly, and mild seizures. The diagnosis of CD was established based on this clinical picture and presence of highly elevated NAA in the urine and was confirmed by molecular testing.

\section{Patient 2}

This one-year-old boy was diagnosed at birth with CD because of a family history. He had repeated aspiration pneumonias during the first year of life and showed severe psychomotor retardation and macrocephaly. From early in his life he had severe difficulties in sucking and was found to have moderate oral phase dysphagia but severe upper pharyngeal dysphagia. He received fundoplication and gastrostomy. His muscle strength in extremities was mildly decreased, i.e., $4 / 5$ and had spasticity. The patient had seizure disorder for which he received Rivotril. The diagnosis of CD was established based on this clinical picture and presence of elevated NAA in the urine and confirmed by molecular testing.

\section{Patient 3}

This boy was diagnosed at 6 months of age with macrocephaly and severe spasticity. He was followed in the clinic for 7 years. The patient presented to our hospital at the age of 2 years with a history of progressive neuroregression of infantile onset, macrocephaly, and seizure disorder. The patient also had a history of recurrent aspiration pneumonia, dental caries, and gastroesophageal reflux for which he had fundoplication and gastrostomy tube insertion. He is severely developmentally delayed, has never vocalized, nor achieved any motor milestones and has remained at a developmental age of 6 months. He developed early and severe spasticity and showed increased deep tendon reflexes. MRI of the brain revealed mild enlargement of posterior fossa and supratentorial CSF spaces indicating diffused cerebellar and cerebral atrophy. Cerebral white matter revealed diffuse abnormal increased signal intensity sparing the internal capsules and the corpus callosum. Central areas of the cerebellar white hemispheres, globi pallidi, and the thalami were all severely involved. MR spectroscopy revealed increased $\mathrm{N}$-acetyl-aspartate peak in conjunction with decreased choline and creatine peaks. Currently, the patient is 10 years old, is wheel chair bound, and has static encephalopathy with no visual fixation, spastic quadriparesis, and central hypotonia. His head circumference is $60 \mathrm{~cm}$ (above the $95 \%$ centile for age). He has seizure disorder and is on antiseizure medications. The diagnosis of $\mathrm{CD}$ was established based on this clinical picture and presence of elevated NAA in the urine and confirmed by molecular testing. A brother presumed to have the same disease died at 18 months of age.

\section{Patient 4}

At 4 months of age after normal delivery, the parents noted delayed development, failure to control head movements, no eye contact, and gross macrocephaly in the patient and his twin brother. Initially, both boys had central hypotonia and widely open anterior fontanel. By 11 months of age, both started to 
show fluctuating muscle tone, with periods of severe spasticity of upper and lower extremity. Neither patient developed gross or fine motor control. They both had repeated aspiration and had to receive oxygen therapy at home. The diagnosis of CD was established in both twins based on this clinical picture and the presence of highly elevated NAA in the urine and confirmed by molecular testing for only Patient 4 .

\section{Patient 5}

This male patient developed neonatal seizures at 20 days of age and from then on achieved no developmental milestones. His parents were first cousins. The patient presented to our hospital at the age of 8 months with neonatal onset seizures, progressive neuroregression, and macrocephaly. The seizures were tonic with fixed gazing or blinking of the eyes. At 8 months he had no head control with severe head-lag, no traction response, no response to visual threat, could not roll over, and never smiled. He had had difficulty swallowing since 4-weeks-of age. He was treated with Carbamazepine. $\mathrm{He}$ started to babble at 2 years and showed some head control at 3 years of age. At 5 years of age he is wheel chair bound and macrocephalic with spastic quadriparesis and central hypotonia. There is no visual contact and flash visual-evoked potentials are absent bilaterally. The diagnosis of $\mathrm{CD}$ was established based on this clinical picture and presence of highly elevated NAA in the urine and confirmed by molecular testing.

\section{Mutation analysis}

Each exon and exon/intron boundary of ASPA were screened by direct sequencing. In Patient 1 , a previously reported homozygous splice donor site mutation (IVS4 + $1 \mathrm{G}>\mathrm{T}$ ) was identified. ${ }^{28}$ Patient 2 was homozygous for the missense mutation, G27R. ${ }^{11}$ Heterozygous status of obligate and other carriers in consented members of the associated families was also confirmed.

Initially in Patients 3 and 4, we were unable to amplify the region encompassing exon 3 of ASPA using PCR. Similarly, amplification of exons 1, 2, 3, 4, 5, and 6 of the ASPA gene in Patient 5 was also not possible. To detect prospective large deletions and the associated breakpoints, we used a combination of MLPA assay, long-PCR, and aCGH. For Patients 3 and 4 , a long-PCR strategy was designed in which the primers spanned from introns 2 to 3 and encompassed exon 3. Combined results from long PCR (Supplemental Fig. 1A), MLPA (Supplemental Fig. 1B), and subsequent data analysis confirmed the presence of a novel large deletion in Patients 3 and 4. Consequently, we designed PCR primers targeting different parts of introns 2 and 3 separately and performed standard PCR experiments to narrow down the breakpoints. The results of these experiments allowed us to pinpoint the precise position of breakpoints in Patients 3 and 4 (Supplemental Fig. 1C). The deletion starts in intron 2 (chr17:3,332,550 position) and ends at intron 3 (chr17: 3,335,897 position) encompassing a $3346 \mathrm{bp}$ region. The region encompassing exon 3 on 3 -D structure of ASPA is highlighted on the recently published x-ray crystal structure of human ASPA (Supplemental Fig. 1D). ${ }^{5}$ The carrier status of the consented family members was also determined (Supplemental Fig. 1A).

Finally, in Patient 5, none of the six exons of ASPA could be successfully amplified using the standard PCR methodology. Recently, there have been reports detailing the presence of gross deletions for this gene and this raised the possibility of a similar gross deletion in this patient. Results of MLPA on this patient confirmed the presence of such a deletion encompassing all six exons of ASPA and beyond. Therefore, aCGH experiments using Agilent's 244K Human Genome CGH Microarray Kit were performed on this patient's DNA to detect the breakpoints. The aCGH results confirmed the presence of a large deletion and its approximate breakpoints (Supplemental Fig. 2). To further characterize the deletion, we designed primers covering the exon/intron boundaries and associated exons for the neighboring gene, TRPV3. The deletion extended to the exon 1 of TRPV 3 and size of the deletion is approximately 92 $\mathrm{kb}$. After performing PCRs using several intronic primers spanning different regions of intron 1 , the deletion was narrowed down to the breakpoint, which was nearly $4.2 \mathrm{~kb}$ far away downstream the exon 1 of TRPV3. Therefore, we concluded that this large deletion is the same deletion as the recently reported large deletion in two patients, ${ }^{13}$ where the proximal deletion point was $7.16 \mathrm{~kb}$ upstream the ASPA start site and the distal deletion point extended to the $3^{\prime}$ sequence of the adjacent TRPV3 gene resulting in deletion of exons 2-17.

\section{Genetic signature, functional annotation, and gene enrichment analysis}

Because $\mathrm{CD}$ is a rare disease and as fibroblasts were only available from two patients, RNA samples on each patient were run on duplicate arrays (technical replicates) and two control RNAs from the control fibroblasts (reference), for a total of eight microarrays. Overall, of 33,096 probes, an average of 23,780 probes were expressed ("present") based on the manufacturer's recommendation of signal to noise ratio exceeding 3 , a value that indicates a $99.9 \%$ confidence that the signal is above background. The dataset showed high correlation within patients and control (technical replicates), and between the patients; with all correlations $>0.95$.

The differentially expressed genes specific to $\mathrm{CD}$ were obtained by overlapping genes lists, i.e., common among the differentially expressed genes for each patient compared with the normal control. Compared with normal fibroblasts (control), 1440 genes were commonly significantly modulated (1116 down-regulated [78\%] and 324 up-regulated [22\%]) in both $\mathrm{CD}$ patients (with absolute fold change $\geq 4$ ). The hierarchical clustering of samples and genes is shown in Supplemental Figure 3 (the top 100 genes commonly dysregulated in both patients were chosen to provide a readable list in the figure). There were more genes with decreased expression than genes with increased expression. Therefore, we selected the genes in proportion to decreased (78\%) and increased (22\%) expression in CD for visualization (Supplemental Fig. 3). Samples are denoted in columns and genes are denoted in rows. The expression level of each gene across the samples is scaled to $[-3$, 
3] interval. These mapped expression levels are depicted using a color scale as shown at the top of the figure, as such highly expressed genes are indicated in red, intermediate in black, and weakly expressed in green. From the differentially expressed genes in both $\mathrm{CD}$ patients, we identified 34 genes and their functional categories, including cell growth/signal transduction/neurogenesis, aspartate/glutamate transporter, apoptosis and inflammation, muscle contraction and development, and compare with the previous study performed by Surendran et al. ${ }^{16-18}$ in the brain of the knockout mouse for CD (Table 1). Among others, neuropilin 1, which plays a role in axon guidance and cell survival, ${ }^{29-31}$ was strongly induced in CD patients. GABA(A) receptor-associated protein (GABARAP), baculoviral IAP repeat-containing 1 (BIRC1), serpine peptidase inhibitor, clade B (ovalbumin) member 2 and 7 (SERPINB2 and SERPINB7), bradykinin receptor B2 (BDKRB2), and capping protein (actin filament) muscle Z-line, alphal were also found to be dysregulated in CD patients. Surendran et al. ${ }^{16-18}$ also reported similar observations in CD mouse such as GABA receptor activity (GABRA6), neurogenic differentiation factor (NEUROD1), serine-type endopaptidase inhibitor (Spi2), and caspase-11 (apoptosis).

To identify the biological processes, molecular functions, and pathways activated by the disease, we used DAVID Bioinformatics Resources, ${ }^{24}$ EASE software, ${ }^{25}$ and the PANTHER Classification system. ${ }^{26}$ By using these bioinformatics tools, we were able to gain greater biological insights into functional processes activated or repressed by the disease much more efficiently and reliably than we could by using the list of differentially expressed genes. ${ }^{32-34}$ We identified overrepresented gene categories among the differentially expressed genes changing $>4$-fold with the disease. We found that genes involved in "cell growth and/or maintenance," "lysosome," "cell proliferation," "Golgi apparatus," "metabolism," "actin binding," and "TGFbeta receptor signaling pathway" were significantly overrepresented (with EASE score >0.02) in differentially expressed genes (data not shown). The PANTHER Classification system determined that genes related to "Protein modification," "Intracellular signaling cascade," "Cell structure and motility," "Actin binding cytoskeletal protein," "Signal transduction," "Mitosis," "Nonmotor and motor actin binding proteins," "Cell cycle," "Kinase modulator," "Developmental processes," "Translation factor," "Chaperone," and "Apoptosis" were the most significantly overrepresented $\left(P<10^{-3}\right)$.

Table 2 shows the top 10 most significantly modulated pathways identified for the 1440 differentially expressed genes that were related to inflammation mediated by chemokine and cytokine signaling pathway $\left(P=3.60 \times 10^{-7}\right)$, angiogenesis $\left(P=5.61 \times 10^{-7}\right)$, FGF signaling pathway $\left(P=1.83 \times 10^{-6}\right)$, apoptosis signaling pathway $\left(P=5.83 \times 10^{-5}\right)$, muscarinic acetylcholine receptor 2 and 4 signaling pathway $(P=1.64 \times$ $\left.10^{-4}\right)$, and Wnt signaling pathway $\left(P=2.67 \times 10^{-4}\right)$. These analyses results not only validate our transcriptome analysis when compared with the candidate gene analysis already reported in the mouse but more importantly, shed new light into a large number of biological processes that take place in human CD.

\section{Confirmation of microarray results using real-time RT-PCR}

Because the validation experiments to confirm the ABI 1700 microarray experiments using TAGMAN and SYBR Green assays were performed by different groups previously and high concordance were reported, and our results were in concordance with the previous knockout studies, we did not perform real-time RTPCR experiments to confirm microarray studies. ${ }^{35-37}$

\section{DISCUSSION}

We screened CD patients from five different families from Saudi Arabia to identify likely mutations in the ASPA gene. We identified two novel large deletions and two previously known mutations (a splice donor-site mutation IVS4 $+1 \mathrm{G}>\mathrm{T}$ and a missense mutation, G27R) in the patients. We also performed global gene expression profiling on the fibroblasts of patients and control samples using ABI Human Genome Survey V.2 arrays. Compared with normal (control) samples, 1440 genes were significantly modulated (up or down) in the Canavan patients (absolute fold change $[\mathrm{FC}] \geq 4$ ). Even though the Patient 5 had the TRPV3 deletion, the expression abnormalities that are discussed were concordant in the two patients thus minimizing the possibility for TRPV3 deficiency interference. Global gene expression profiling results indicated that some genes, involved in apoptosis, muscle contraction and development, mitochondrial oxidation, inflamation and glutamate, and aspartate metabolism, were significantly dysregulated.

Patient 1 has a homozygous splice donor-site mutation, that IVS4 $+1 \mathrm{G}>\mathrm{T}$, is similar to that described by Rady et al. ${ }^{28}$ in a Turkish family. They used the sequencing technique and showed a G>T substitution at the donor site of intron-4, which resulted in a GT $\rightarrow$ TT change in the patient.

The mutation of Patient 2 was G27R, first reported by Kaul et al. ${ }^{11}$ The mutation is located in a region of the hydrophobic core of the enzyme. G27R is likely to cause local unfolding problems due to the positioning of a massive, charged residue into the core and could conceivably cause a severe phenotype. Because the mutation is likely to result in an unstable protein, it possibly undergoes degradation in vivo. ${ }^{5}$ The effect of the mutation on the enzyme activity was tested by in vitro mutagenesis and expression of mutant cDNA in COS1 cells. G27R mutant ASPA showed a reduced residual activity $(\sim 3 \%)$ compared with the native enzyme. ${ }^{11}$

The mutation found in Patients 3 and 4 is a novel deletion extending from introns 2 and 3 comprising the full exon 3 and extending $3346 \mathrm{bp}$. The presence of such a deletion is likely to result in an enzyme having affected zinc coordination, substrate binding, and local unfolding problems eventually leading to an unstable protein that will result in degradation in vivo. ${ }^{5}$

In Patient 5, none of the six exons of ASPA could be successfully amplified using the standard PCR methodology. Using MLPA and aCGH techniques, we confirmed the presence of recently reported large deletion $(92 \mathrm{~kb})$ in Patient 5. Previously, 
Table 1

Identification of 34 genes induced in our CD patients and comparison with CD knock-out mouse study by Surendran et al. ${ }^{16-18}$

\begin{tabular}{|c|c|c|c|c|c|}
\hline \multirow[b]{2}{*}{ Functional category } & \multicolumn{3}{|c|}{ Significant genes in human CD patients } & \multicolumn{2}{|c|}{ CD knockout mouse } \\
\hline & Gene name & P1 FC & $\mathrm{P} 2 \mathrm{FC}$ & Gene & FC \\
\hline \multicolumn{6}{|c|}{ Cell growth/signal transduction/neurogenesis } \\
\hline GABARAP & GABA(A) receptor-associated protein & $5.9 \downarrow$ & $2.9 \downarrow$ & Gabra6 & $119.1 \downarrow$ \\
\hline GABARAPL2 & GABA(A) receptor-associated protein-like 2 & $3.8 \downarrow$ & $3.5 \downarrow$ & & \\
\hline CGRRF1 & Cell growth regulator with ring finger domain 1 & $7.3 \downarrow$ & $4.5 \downarrow$ & & \\
\hline$N R P 1$ & Neuropilin 1 & $61 \downarrow$ & $43 \downarrow$ & Neurod1 & $7.0 \downarrow$ \\
\hline NTN4 & Netrin 4 & $19 \downarrow$ & $11 \downarrow$ & & \\
\hline HOXD10 & Homeobox D10 & $14 \downarrow$ & $17.5 \downarrow$ & & \\
\hline SPAST & Spastin & $14 \downarrow$ & $19.9 \downarrow$ & & \\
\hline FOXF2 & Forkhead box F2 & $11 \downarrow$ & $4.7 \downarrow$ & & \\
\hline ROBO1 & Roundabout, axon guidance receptor, homolog 1 & $11 \downarrow$ & $8.7 \downarrow$ & & \\
\hline NEGR1 & Neuronal growth regulator 1 & $5.2 \downarrow$ & $9.5 \downarrow$ & & \\
\hline FGFR1 & fibroblast growth factor receptor 1 & $33 \downarrow$ & $36 \downarrow$ & & \\
\hline HIF1A & Hypoxia-inducible factor 1, alpha subunit & $21 \downarrow$ & $14 \downarrow$ & & \\
\hline EFNB2 & Ephrin-B2 & $13 \downarrow$ & $15 \downarrow$ & & \\
\hline PTPRS & Protein tyrosine phosphatase, receptor type, $S$ & $9.8 \downarrow$ & $4.7 \downarrow$ & & \\
\hline GMFB & Glia maturation factor, beta & $8.0 \downarrow$ & $6.4 \downarrow$ & & \\
\hline NLGN1 & Neuroligin 1 & $6.8 \downarrow$ & $5.7 \downarrow$ & & \\
\hline \multicolumn{6}{|c|}{ Aspartate/glutamate transporter } \\
\hline SLC16A7 & $\begin{array}{l}\text { Solute carrier family } 16 \text { (monocarboxylic acid } \\
\text { transporters), member } 7\end{array}$ & $6.8 \downarrow$ & $6.8 \downarrow$ & Eaat4 & $9.7 \downarrow$ \\
\hline SLC1A6 & $\begin{array}{l}\text { Solute carrier family } 1 \text { (high affinity aspartate/glutamate } \\
\text { transporter), member } 6\end{array}$ & $2.2 \downarrow$ & $1.6 \downarrow$ & & \\
\hline \multicolumn{6}{|c|}{ Apoptosis and inflammation } \\
\hline SERPINB7 & Serpin peptidase inhibitor, clade B (ovalbumin), member 7 & $7.8 \uparrow$ & $7.4 \uparrow$ & Spi2 & $29.8 \uparrow$ \\
\hline SERPINA4 & $\begin{array}{l}\text { Serpin peptidase inhibitor, clade A (alpha-1 antiproteinase, } \\
\text { antitrypsin), member } 4\end{array}$ & $3.0 \uparrow$ & $1.5 \uparrow$ & & \\
\hline$E L A 2 B$ & Elastase 2B & $3.0 \uparrow$ & $3.0 \uparrow$ & & \\
\hline$B I R C 1$ & Baculoviral IAP repeat-containing 1 & $9 \uparrow$ & $6 \uparrow$ & & \\
\hline HTRA3 & HtrA serine peptidase 3 & $6 \uparrow$ & $5 \uparrow$ & & \\
\hline PDCD11 & Programmed cell death 11 & $3.5 \uparrow$ & $3.3 \uparrow$ & & \\
\hline SERPINB2 & Serpin peptidase inhibitor, clade B (ovalbumin), member 2 & $408 \downarrow$ & $164 \downarrow$ & & \\
\hline TNFRSF19 & tumor necrosis factor receptor superfamily, member 19 & $62 \downarrow$ & $45 \downarrow$ & & \\
\hline CASP4 & Caspase 4 , apoptosis-related cysteine peptidase & $2 \uparrow$ & $5 \uparrow$ & Caspase-11 & $4.4 \uparrow$ \\
\hline$C L U$ & Clusterin & $4.8 \uparrow$ & $3.8 \uparrow$ & & \\
\hline$I L 7 R$ & Interleukin 7 receptor & $7.3 \uparrow$ & $5 \uparrow$ & $\begin{array}{l}\text { IL- } 1 \beta \text { converting } \\
\text { enzyme }\end{array}$ & $3.8 \uparrow$ \\
\hline IL11 & Interleukin 11 & $10 \uparrow$ & $10.6 \uparrow$ & & \\
\hline \multicolumn{6}{|c|}{ Muscle contraction and development } \\
\hline$B D K R B 2$ & Bradykinin receptor B2 & $59 \downarrow$ & $20 \downarrow$ & & \\
\hline$M B N L 2$ & Muscleblind-like 2 & $39 \downarrow$ & $31 \downarrow$ & & \\
\hline$A D D 3$ & Adducin 3 (gamma) & $47 \downarrow$ & $21 \downarrow$ & & \\
\hline CAPZA1 & Capping protein(actin filament) muscle Z-line, alphal & $146 \downarrow$ & $19 \downarrow$ & & \\
\hline
\end{tabular}


Table 2

Top 10 significantly over-represented pathways among genes differentially expressed in CD patients

\begin{tabular}{lcc}
\hline PANTHER pathway & No. genes $^{a}$ & $P^{b}$ \\
\hline $\begin{array}{l}\text { Inflammation mediated by chemokine } \\
\text { and cytokine signaling pathway }\end{array}$ & 46 & $3.60 \mathrm{E}-07$ \\
Angiogenesis & 37 & $5.61 \mathrm{E}-07$ \\
FGF signaling pathway & 27 & $1.83 \mathrm{E}-06$ \\
VEGF signaling pathway & 19 & $2.59 \mathrm{E}-06$ \\
Integrin signaling pathway & 35 & $4.48 \mathrm{E}-06$ \\
PDGF signaling pathway & 28 & $3.50 \mathrm{E}-05$ \\
$\begin{array}{l}\text { Cytoskeletal regulation by Rho } \\
\text { GTPase }\end{array}$ & 22 & $4.42 \mathrm{E}-05$ \\
Apoptosis signaling pathway & & \\
Muscarinic acetylcholine receptor 2 & 24 & $5.83 \mathrm{E}-05$ \\
$\quad$ and 4 signaling pathway & 13 & $1.64 \mathrm{E}-04$ \\
Wnt signaling pathway & 42 & $2.67 \mathrm{E}-04$ \\
\hline
\end{tabular}

The number of differentially expressed genes belonging to each pathway is shown.

${ }^{a}$ The number of genes among the differentially expressed genes in respective pathways.

${ }^{b}$ PANTHER used the binomial test to determine biological processes or molecular functions overrepresented among genes differentially expressed. $P$ values for each category were calculated from the binomial test statistic. Significance is defined as $P<0.05$.

the deletion was found in two patients; Italian and German/ British, respectively. ${ }^{13}$ The TRPV3 gene belongs to a family of nonselective cation channels. Its function is unknown; however, it has been implicated in temperature sensation and vasoregulation. This gene is in close proximity to another family member TRPV1, which is a receptor for capsaicin. The neurologically terminal patient in this study could not be tested for temperature sensation.

We compared our gene expression results found in the patients with those findings previously reported..$^{7,16-18,38-41}$ The genetic signature studies indicated significant similarities between our study and those found in the CD mouse model. The genes related to apoptosis/antiapoptosis, cell growth, signal transduction genes, cell death, and inflammatory genes were significantly dysregulated as reported in CD mouse brain. ${ }^{18}$ In fact our analysis of biological pathways represented by the differentially expressed genes in CD patients revealed that "inflammation mediated by chemokine and cytokine signaling pathways" and "apoptosis signaling pathways" were the first and sixth most significantly overrepresented pathways. Surprising findings in our study included dysregulation of the genes related to muscle development and contraction, cytoskeleton-related genes, apoptosis, and mitochondrial activity-related genes.

The genes related to apoptosis/antiapoptosis activities such as BIRC1 and HTRA3 were upregulated in the patients compared with the normals, whereas SERPINB2, TNFRSF19, $S O C S 3$, and $R H O B$ were significantly downregulated in $C D$ patients. It has been reported that BIRC1 is linked to spinal muscular atrophy, which occurs in children with degeneration of the motor neurons and leads to progressive muscular weakness and atrophy..$^{42} B I R C 1$ was also proposed to participate in antiapoptosis pathways together with some of the caspases such as CASP3, CASP1, and CASP9. DFFA that triggers DNA fragmentation and functions together with $C A S P 3$ was also decreased 4 -fold. ${ }^{43,44}$

Surendran et al. ${ }^{17,18,40}$ suggest that the abnormal expression of some genes such as the GABA-A receptor GABRA6, and neurogenic differentiation factor may have some effect on hypotonia and muscle weakness. It was also suggested that these observations needed to be documented in humans. It is interesting to find that some of the related genes were significantly affected in Saudi CD patients. The genes possibly related to muscle weakness and muscle contraction, such as $N E B L$, MBNL2, SNTB1, BDKRB2, ASPH, PRKG1 and SVIL, were significantly downregulated in our patients. Some other genes found to be affected and related to muscle contraction and development and myosin activities were MYBPHL, GAL, GLMN2, GSR, MYH2, and MYH11. These observations support muscle-related functions being significantly affected. In fact, the functional category enrichment analysis of the differentially expressed genes also identified "cell structure and motility" and "actin binding cytoskeletal protein" as being significantly enriched.

Significant mRNA changes related to mitochondrial function and oxidoreductase activities were also observed. It is well established that mitochondrial morphology is different in CD. In this regard, $P P R C 1$, a gene responsible for mitochondrial biogenesis was increased whereas $N R F 1$, a nuclear respiratory factor, directly interacting with $P P R C 1$ to activate mitochondrial biogenesis was downregulated. The mRNA of genes that import various compounds into mitochondria, such as MTX1, SLC25A32, SLC25A24, and MCFP, were affected in different manners.

The mRNAs of genes that directly influence oxidative phosphorylation such as SDHA, SDHAL2, Citrate synthase, FECH, and F1 subunit of $A T P A F 1$ were significantly decreased. In a key step in mitochondrial ATP synthesis, the F1 subunit of ATPAF1 acts like a maintenance gene and is required for the assembly of beta (F(1)-beta) and alpha (F(1)-alpha) subunits into mitochondrial ATPase. These mRNA changes were paralleled by changes in the mRNA of a large number of oxidoreductases; some of which are located in the mitochondria, others being in the cytosol and endoplasmic reticulum. The list includes short chain acyl-CoA dehydrogenase, LOXL2, $Z A D H 2, A K R 1 C 1, G S R$, and $A D H 1 B$. It is interesting to note that a subunit of Cytochrome P450 (CYP51A1), the main detoxifying enzyme, was severely decreased.

Glutamate- and aspartate-related genes such as $G P R C 5 B$, GLUL, PGCP, PCMTD1, and ASPH were greatly dysregulated suggesting that glutamate and aspartate metabolic pathways are likely to be affected in these patients. The mRNA of GPR51, GABARAPL2, and GPRC5B that are related to GABA activity were also decreased whereas similar genes such as GABRE, GPRC5C, and GABBR1 were upregulated. In fact, the "metabotropic glutamate receptor group II and group III pathways" 
and "glutamine glutamate conversion pathway" were among the significantly impacted pathways represented by the differentially expressed genes in CD patients.

The mRNA of clusterin was also increased around 4-fold in the Saudi CD patients; this protein binds extracellular proteins and targets them for endocytosis and degradation. However, expression of various metalloproteases such as some of the ADAM group was significantly decreased. MAP7 that promotes microtubule stability was also downregulated.

The mRNA of ATF5 essential for neuronal differentiation was increased significantly whereas that of HAND2 another morphogenesis protein was decreased considerably. The mRNAs of 68 genes related to various aspects of nucleic acid metabolism were variably altered.

Because we found critically modulated genes implicated in mitochondrial function, mtDNA sequencing was performed according to standard methods. No point mutations and deletions were detected for the following mitochondria-associated diseases: MELAS, MERRF, NARP, cardiomyopathies, LHON, Kearns-Sayre, CPEO, and Pearson Marrow/Pancreas Syndrome. Therefore, association of any of these diseases to Canavan was excluded.

In summary, we have screened Saudi CD patients from five families for putative mutations and identified four mutations including two novel large deletions. Our whole genome gene expression analysis indicated that the activities related to apoptosis, inflammation, cell death, mitochondrial process, protein metabolism, muscle development and contraction, and cytoskeleton-related activities were critically affected. Therefore, mitochondrial oxidative phosphorylation and drug detoxification should be impaired. Because we sequenced complete mtDNA and found no point mutations or deletions, the changes related to mitochondrial activities should be related to CD but not to any known mitochondrial diseases. Also, our results shed some light on questions related to the presence of muscle weakness and hypotonia in CD patients by indicating that the genes related to cell motility, muscle contraction and development, actin binding, and cytoskeletal-related activities were significantly dysregulated. Overall, these observations are in accordance with previous studies performed in a knockout mouse model. To the best of our knowledge, this is the first genome-wide gene expression study performed on CD patients' samples and should shed further light on the pathogenesis of this disease in humans.

\section{ACKNOWLEDGMENTS}

This study was supported by Prince Salman Center for Disability Research.

The authors thank KFSHRC Research Advisory Council for the approval of the project and Dr. Ghazi Alsbeih for providing the control fibroblasts.

\section{References}

1. Matalon R, Michals K, Sebesta D, Deanching M, Gashkoff P, Casanova J. Aspartoacylase deficiency and $\mathrm{N}$-acetylaspartic aciduria in patients with Canavan disease. Am J Med Genet 1988;29:463-471.
2. Banker BQ, Robertson JT, Victor M. Spongy degeneration of the central nervous system in infancy. Neurology 1964;14:981-1001.

3. Ozand PT, Gascon GG, Dhalla M. Aspartoacylase deficiency and Canavan disease in Saudi Arabia. Am J Med Genet 1990;35:266-268.

4. Kaul R, Gao GP, Balamurugan K, Matalon R. Cloning of the human aspartoacylase cDNA and a common missense mutation in Canavan disease. Nat Genet 1993;5: $118-123$.

5. Bitto E, Bingman CA, Wesenberg GE, McCoy JG, Phillips GN Jr. Structure of aspartoacylase, the brain enzyme impaired in Canavan disease. Proc Natl Acad Sci USA 2007;104:456-461.

6. Herga S, Berrin JG, Perrier J, Puigserver A, Giardina T. Identification of the zinc binding ligands and the catalytic residue in human aspartoacylase, an enzyme involved in Canavan disease. FEBS Lett 2006;580:5899-5904.

7. Surendran S, Shihabuddin LS, Clarke J, et al. Mouse neural progenitor cells differentiate into oligodendrocytes in the brain of a knockout mouse model of Canavan disease. Brain Res Dev Brain Res 2004;153:19-27.

8. Hershfield JR, Pattabiraman N, Madhavarao CN, Namboodiri MA. Mutational analysis of aspartoacylase: implications for Canavan disease. Brain Res 2007;1148: $1-14$.

9. Janson CG, Kolodny EH, Zeng BJ, Raghavan S, et al. Mild-onset presentation of Canavan's disease associated with novel G212A point mutation in aspartoacylase gene. Ann Neurol 2006;59:428-431.

10. Kaul R, Gao GP, Aloya M, et al. Canavan disease: mutations among Jewish and non-Jewish patients. Am J Hum Genet 1994;55:34-41.

11. Kaul R, Gao GP, Matalon R, et al. Identification and expression of eight nove mutations among non-Jewish patients with Canavan disease. Am J Hum Genet 1996; 59:95-102.

12. Zeng BJ, Pastores GM, Leone P, et al. Mutation analysis of the aspartoacylase gene in non-Jewish patients with Canavan disease. Adv Exp Med Biol 2006;576:165-173; discussion 361-163.

13. Zeng BJ, Wang ZH, Torres PA, et al. Rapid detection of three large novel deletions of the aspartoacylase gene in non-Jewish patients with Canavan disease. Mol Genet Metab 2006;89:156-163.

14. Baslow $\mathrm{MH}$. Brain $\mathrm{N}$-acetylaspartate as a molecular water pump and its role in the etiology of Canavan disease: a mechanistic explanation. JMol Neurosci 2003;21:185-190.

15. Namboodiri AM, Peethambaran A, Mathew R, et al. Canavan disease and the role of $\mathrm{N}$-acetylaspartate in myelin synthesis. Mol Cell Endocrinol 2006;252:216-223.

16. Surendran S, Matalon KM, Szucs S, Tyring SK, Matalon R. Metabolic changes in the knockout mouse for Canavan's disease: implications for patients with Canavan's disease. J Child Neurol 2003;18:611-615.

17. Surendran S, Michals-Matalon K, Quast MJ, et al. Canavan disease: a monogenic trait with complex genomic interaction. Mol Genet Metab 2003;80:74-80.

18. Surendran S, Rady PL, Michals-Matalon K, et al. Expression of glutamate transporter, GABRA6, serine proteinase inhibitor 2 and low levels of glutamate and GABA in the brain of knock-out mouse for Canavan disease. Brain Res Bull 2003; 61:427-435.

19. Rieder MJ, Taylor SL, Tobe VO, Nickerson DA. Automating the identification of DNA variations using quality-based fluorescence re-sequencing: analysis of the human mitochondrial genome. Nucleic Acids Res 1998;26:967-973.

20. Brandon MC, Lott MT, Nguyen KC, et al. MITOMAP: a human mitochondria genome database-2004 update. Nucleic Acids Res 2005;33(Database issue):D611D613.

21. Sellner LN, Taylor GR. MLPA and MAPH: new techniques for detection of gene deletions. Hum Mutat 2004;23:413-419.

22. Gentleman RC, Carey VJ, Bates DM, et al. Bioconductor: open software development for computational biology and bioinformatics. Genome Biol 2004;5:R80.

23. Jeffery IB, Higgins DG, Culhane AC. Comparison and evaluation of methods for generating differentially expressed gene lists from microarray data. BMC Bioinformatics 2006;7:359.

24. Huang DW, Sherman BT, Tan Q, et al. The DAVID Gene Functional Classification Tool: a novel biological module-centric algorithm to functionally analyze large gene lists. Genome Biol 2007;8:R183.

25. Hosack DA, Dennis G Jr, Sherman BT, Lane HC, Lempicki RA. Identifying biolog ical themes within lists of genes with EASE. Genome Biol 2003;4:R70.

26. Thomas PD, Campbell MJ, Kejariwal A, et al. PANTHER: a library of protein families and subfamilies indexed by function. Genome Res 2003;13:2129-2141.

27. Thomas PD, Kejariwal A, Guo N, et al. Applications for protein sequence-function evolution data: mRNA/protein expression analysis and coding SNP scoring tools. Nucleic Acids Res. 2006;34(Web Server issue):W645-W650.

28. Rady PL, Penzien JM, Vargas T, Tyring SK, Matalon R. Novel splice site mutation of aspartoacylase gene in a Turkish patient with Canavan disease. Eur J Paediatr Neuro 2000;4:27-30.

29. He Z, Tessier-Lavigne M. Neuropilin is a receptor for the axonal chemorepellent Semaphorin III. Cell 1997;90:739-751. 


\section{Kaya et al.}

30. Cackowski FC, Xu L, Hu B, Cheng SY. Identification of two novel alternatively spliced Neuropilin-1 isoforms. Genomics 2004;84:82-94.

31. Hu B, Guo P, Bar-Joseph I, et al. Neuropilin-1 promotes human glioma progression through potentiating the activity of the HGF/SF autocrine pathway. Oncogene 2007; 26:5577-5586.

32. Danley PD, Mullen SP, Liu F, Nene V, Quackenbush J, Shaw KL. A cricket Gene Index: a genomic resource for studying neurobiology, speciation, and molecular evolution. BMC Genomics 2007;8:109.

33. Quackenbush J. Extracting biology from high-dimensional biological data. J Exp Biol 2007;210(Pt 9):1507-1517.

34. Song JS, Maghsoudi K, Li W, Fox E, Quackenbush J, Shirley Liu X. Microarray blob-defect removal improves array analysis. Bioinformatics (Oxford, England). 2007;23:966-971.

35. Barbacioru CC, Wang Y, Canales RD, et al. Effect of various normalization methods on Applied Biosystems expression array system data. BMC Bioinformatics 2006;7:533.

36. Sorlie T, Wang Y, Xiao C, et al. Distinct molecular mechanisms underlying clinically relevant subtypes of breast cancer: gene expression analyses across three different platforms. BMC Genomics 2006;7:127.

37. Walker SJ, Wang Y, Grant KA, Chan F, Hellmann GM. Long versus short oligonucleotide microarrays for the study of gene expression in nonhuman primates. J Neurosci Methods 2006;152:179-189.
38. Surendran S, Campbell GA, Tyring SK, Matalon R. Aspartoacylase gene knockout results in severe vacuolation in the white matter and gray matter of the spinal cord in the mouse. Neurobiol Dis 2005;18:385-389.

39. Surendran S, Ezell EL, Quast MJ, et al. Aspartoacylase deficiency does not affect $\mathrm{N}$-acetylaspartylglutamate level or glutamate carboxypeptidase II activity in the knockout mouse brain. Brain Res 2004;1016:268-271.

40. Surendran S, Ezell EL, Quast MJ, et al. Mental retardation and hypotonia seen in the knock out mouse for Canavan disease is not due to succinate semialdehyde dehydrogenase deficiency. Neurosci Lett 2004;358:29-32.

41. Surendran S, Matalon KM, Tyring SK, Matalon R. Molecular basis of Canavan's disease: from human to mouse. J child Neurol 2003;18:604-610.

42. Roy N, Mahadevan MS, McLean M, et al. The gene for neuronal apoptosis inhibitory protein is partially deleted in individuals with spinal muscular atrophy. Cell 1995; 80:167-178

43. Damiano JS, Oliveira V, Welsh K, Reed JC. Heterotypic interactions among NACHT domains: implications for regulation of innate immune responses. Biochem J 2004 381(Pt 1):213-219.

44. Davoodi J, Lin L, Kelly J, Liston P, Mackenzie AE. Neuronal apoptosis-inhibitory protein does not interact with Smac and requires ATP to bind caspase-9. J Biol Chem 2004;279:40622-40628 